\title{
QUALIDADE DE VIDA EM DEGLUTIÇÃO E CÂNCER DE CABEÇA E PESCOÇO: REVISÃO DE LITERATURA
}

\author{
SWALLOWING QUALITY OF LIFE AND HEAD \\ AND NECK CANCER: LITERATURE REVIEW
}

\section{Raquel de Santana Oliveira Marques', Agda Santana Costa', Alena Ribeiro Alves Peixoto Medrado ${ }^{2}$, Gabriela Botelho Martins ${ }^{3}$, Hayana Ramos Lima ${ }^{4}$, Manoela Carrera ${ }^{5}$}

\begin{abstract}
Autora para correspondência: Manoela Carrera - manoelacarrera@gmail.com 'Graduanda de Fonoaudiologia na Universidade do Estado da Bahia. Salvador, Bahia, Brasil. ${ }^{2}$ Doutora em Patologia Humana. Professora na Escola Bahiana de Medicina e Saúde Pública e na Universidade Federal da Bahia. Salvador, Bahia, Brasil. ${ }^{3}$ Doutora em Estomatologia Clínica. Professora na Universidade Federal da Bahia. Salvador, Bahia, Brasil. ${ }^{4}$ Doutora em Ciências Odontológicas Aplicadas, Biologia Oral. Professora na União Metropolitana de Ensino e Cultura - UNIME. Lauro de Freitas, Bahia, Brasil.

${ }^{5}$ Doutora em Estomatopatologia. Professora na Universidade do Estado da Bahia e na Universidade Federal da Bahia. Salvador, Bahia, Brasil.
\end{abstract}

RESUMO I Objetivo: O estudo teve como objetivo revisar a literatura a respeito da qualidade de vida relacionada à deglutição de pacientes com câncer de cabeça e pescoço. Métodos: Buscou-se estudos relatando a fisiologia da deglutição e os aspectos envolvidos na disfagia, como definição, causas e seu impacto na qualidade de vida. Foram utilizados os bancos de dados eletrônicos Pubmed, Medline, Scielo, livros, assim como de artigos identificados nas referências. Resultados: Distúrbios da deglutição podem ocorrer devido à doença e/ou modalidade de tratamento, persistindo durante ou após o término do tratamento. A frequência e gravidade da disfagia dependerão da modalidade de tratamento utilizada, localização e estágio do tumor, quando envolvidos em estruturas que participem da deglutição. Pacientes com tumores em estágios mais avançados e localizados na faringe tendem a apresentar piora na função da deglutição. Tais fatores poderão interferir diretamente na deglutição e qualidade de vida do paciente, sobretudo nas relações familiares, sociais e no estilo de vida. Conclusão: Apesar de não substituir as avaliações clínicas e instrumentais, investigar a qualidade de vida em deglutição pode contribuir para avaliar aspectos específicos sobre $\circ$ bem-estar do paciente que não são contemplados nestas avaliações.

Palavras-chave: Qualidade de vida; Deglutição; Câncer de Cabeça e Pescoço; Disfagia.

\begin{abstract}
Objective: This study aimed to review the literature on the quality of life related to swallowing of patients with head and neck cancer. Methods: We searched for studies describing the physiology of swallowing and aspects involved in dysphagia, such as definition, causes and their impact on quality of life. We used the electronic databases Pubmed, Medline, Scielo, books, as well as articles identified in the references. Results: Deglutition disorders may occur due to disease and / or treatment modality, persisting during or after termination of treatment. The frequency and severity of dysphagia will depend on the modality of treatment used, location and stage of the tumor, when involved in structures that participate in swallowing. Patients with tumors in more advanced stages and located in the pharynx try to present worsening of swallowing function. Such factors may directly affect swallowing and quality of life of the patient, especially in family, social and lifestyle relationships. Conclusion: Although it does not replace clinical and instrumental evaluations, investigating the quality of life in swallowing may contribute to assess specific aspects of patient well-being that are not contemplated.
\end{abstract}

Keywords: Quality of life; Swallowing; Head and Neck Cancer; Dysphagia. 


\section{INTRODUÇÃO}

O câncer é uma doença multifatorial crônica que tem afetado, sobretudo, os países em desenvolvimento. Estima-se que no Brasil a incidência de câncer para o ano de 2016 e 2017 seja de 600 mil casos novos. Na Região Nordeste, o câncer da cavidade oral é o quinto mais frequente em homens e o nono mais frequente em mulheres, quando excluídos os tumores de pele não melanoma. Os principais fatores de risco associados são o estilismo e o tabagismo'.

A disfagia é um sintoma comum em pacientes oncológicos, em especial para aqueles com câncer de cabeça e pescoço devido à proximidade das estruturas que participam da deglutição. Caracteriza-se pela dificuldade em deglutir sólidos ou líquidos, acometendo qualquer fase da deglutição. Pode surgir como decorrência da doença ou da modalidade de tratamento utilizado, ocorrendo durante ou mesmo após o término do tratamento ${ }^{2}$.

Dentre as suas principais complicações estão a desnutrição, desidratação, aspiração do alimento para a via aérea e pneumonia. Tais fatores estão intimamente relacionados com o estado de saúde e qualidade de vida do paciente ${ }^{3}$.

O termo qualidade de vida atualmente tem sido alvo de estudos por considerar aspectos globais do bem estar do indivíduo. É um conceito subjetivo e multidimensional que pode ser influenciado por variáveis como estado físico, psicológico, econômico e sociocultural ${ }^{4}$. A disfagia pode afetar de modo significativo a qualidade de vida devido à mudança de hábitos, destacando-se o prazer ao se alimentar e as relações sociais, visto que a alimentação simboliza aproximação com familiares e amigos ${ }^{5,6}$.

A avaliação do impacto da disfagia sobre a qualidade de vida do paciente oncológico possibilita melhor manejo e intervenção quanto aos aspectos físicos, emocionais e sociais que não são contemplados por meio de avaliações objetivas. Desta forma, o presente estudo teve como objetivo revisar a literatura a respeito da qualidade de vida em deglutição de pacientes com câncer de cabeça e pescoço. Para isso buscou-se definição, causas e mecanismos envolvidos no processo da deglutição e disfagia, bem como aspectos relacionados ao câncer que geram repercussões na qualidade de vida do paciente.

\section{MÉTODO}

A revisão de literatura foi realizada a partir de livros e bancos de dados eletrônicos como Pubmed, Medline e Scielo. Utilizou-se os descritores: "deglutição", "swallowing", "swallowing disorders", "dysphagia oropharyngeal", "swallowing and cancer", "dysphagia and cancer", "head and neck cancer", "head and neck cancer and swallowing", " quality of life cancer".

Os critérios de inclusão foram artigos publicados na íntegra, nos idiomas português e inglês, e livros considerados referência na temática abordada. Foram excluídos artigos que não atendiam a proposta do estudo, relatos de caso e textos de caráter não científico. Após o levantamento quantitativo dos artigos científicos, foi realizada análise qualitativa de acordo com os objetivos deste estudo por meio da sua leitura.

\section{REVISÃO DE LITERATURA}

\section{Deglutição e disfagia}

A deglutição tem como funções a nutrição e a hidratação do indivíduo com a manutenção do estado nutricional e proteção da via aérea, garantindo assim, a sobrevivência ${ }^{7}$.

Didaticamente é dividida em quatro fases, sendo estas intrinsecamente relacionadas, sequenciais e harmônicas ${ }^{8}$. Em síntese, na primeira fase, denominada fase antecipatória, o alimento é moído e misturado com a saliva para que seja formado o bolo alimentar. Na fase seguinte, a fase oral, este 
bolo é posicionado no sulco longitudinal da língua e projetado para a faringe por meio de movimentos anteroposteriores da língua, da atuação dos lábios, mandíbula e músculos. Durante a fase faríngea, há a ativação do reflexo da deglutição, elevação do palato mole, os músculos constritores da faringe se contraem, de superior para inferior, a laringe é elevada e anteriorizada, as pregas vocais encontram-se em adução e há a eversão da epiglote. Por fim, o músculo cricofaríngeo se descontrai para permitir que o bolo alimentar siga para o esôfago. Na fase final, a fase esofágica, o peristaltismo dos músculos esofágicos transporta $\circ$ alimento para $\circ$ estômago ${ }^{9,10}$. Para que funcionem adequadamente, é necessária ação neuromuscular (propriocepção, paladar, sensibilidade, tônus, mobilidade e tensão) associada ao desejo de se alimentar ${ }^{8}$. Em torno de 30 músculos e 6 pares de nervos encefálicos atuam na deglutição, com a participação do córtex cerebral e do tronco encefálico. Os pares de nervos encefálicos são o trigêmeo (V), facial (VII), glossofaríngeo (IX), vago (X), acessório espinal (XI) e hipoglosso $(\mathrm{XII})^{11,12}$.

A palavra disfagia tem origem grega derivada das palavras dys (dificuldade) e phagein (comer). É um sintoma decorrente do atraso ou dificuldade na passagem de sólidos ou líquidos da cavidade oral ao estômago ${ }^{13}$. Pode ocorrer na fase orofaríngea ou esofágica e gerar limitações na função, além de complicações como a aspiração de alimento para a via aérea, pneumonia, desidratação e desnutrição, interferindo na qualidade de vida (QV) do indivíduo em vários aspectos sejam eles emocionais, físicos ou socioculturais ${ }^{3}$.

Dentre as causas da disfagia orofaríngea podem estar doenças neurológicas, como, por exemplo, doença de Parkinson, Esclerose Lateral Amiotrófica, ou alterações estruturais, como o câncer de cabeça e pescoço $(C C P)^{9}$.

Alguns indicadores de problemas de deglutição incluem histórico de dificuldade para deglutir, presença de engasgos/tosse, sensação de bolo na garganta, substituição de consistências alimentares, nutrição deficiente e qualidade vocal anormal depois de deglutir, como por exemplo, a percepção da voz "molhada"2,14. Os distúrbios da deglutição representam um grande impacto na QV pela relação da alimentação com os aspectos nutricionais, sociais e com a manutenção da vida ${ }^{15}$.

Disfagia e câncer

A disfagia é um sintoma comum em pacientes com câncer, porém mais encontrada em pacientes com $\mathrm{CCP}^{8}$. Estes podem queixar-se de disfagia devido à doença em si ou como consequência do tratamento ${ }^{16}$.

Os sintomas para esse tipo de câncer são variados. Além da disfagia podem apresentar dor, rouquidão, odinofagia, tosse e perda de peso. Esses efeitos variam em frequência de ocorrência, severidade e complexidade, podendo permanecer durante e/ou após o término do tratamento oncológico ${ }^{15}$.

Entre os fatores que influenciam na frequência e gravidade da disfagia antes do tratamento e suas sequelas estão o estágio do tumor e a sua localização, desde que estes afetem a motilidade das estruturas envolvidas ${ }^{2,17,18}$. A função da deglutição foi avaliada em 352 pacientes com CCP pré-tratamento. Observou-se que os participantes tiveram maior tempo de trânsito oral e faríngeo, maior quantidade de resíduo na cavidade oral e faringe, menor duração na abertura do músculo cricofaríngeo e, consequentemente, menor eficiência na deglutição. Antes do tratamento, 59\% dos participantes queixavam-se de disfagia. Houve piora significante na deglutição quanto ao estágio do tumor - T1 $(23,1 \%)$, T2 $(25 \%)$, T3 $(44,8 \%)$, T4 $(58,8 \%)$ - e localização. Os pacientes com tumores da cavidade oral foram menos propensos a apresentar queixas (representando $28,2 \%$ ) do que os pacientes com lesões na faringe $(50,9 \%)^{19}$.

Em contrapartida, Cohen e cols. $(2008)^{20}$ investigaram a associação entre melhoria da função de deglutição com o estágio avançado do tumor antes e após a quimioterapia concomitante $e$ radioterapia. Fizeram parte do estudo 95 pacientes com CCP avaliados antes e após 1 e 2 meses do tratamento. Observou-se que os pacientes com câncer de laringe em estágio avançado (T3 e T4) eram menos propensos a ter piora da deglutição após um ciclo de quimioterapia concomitante $e$ radioterapia. A função foi preservada devido ao tratamento, havendo uma melhora após a sua conclusão ${ }^{20}$. 
Os sintomas mais prevalentes identificados, independentemente da modalidade de tratamento, são problemas com muco ou secreções e mucosite, bem como alteração no paladar, xerostomia, dor e fadiga $^{21}$.

Estudos demonstram que tratamentos mais agressivos utilizados em doença avançada interferem significativamente nos hábitos alimentares, nutrição e QV do paciente. A prevalência da disfagia orofaríngea foi avaliada a longo prazo em pacientes com CCP tratados com cirurgia e radioterapia ou quimiorradioterapia $^{22}$. $O$ impacto da disfagia em hábitos alimentares, estado nutricional e $Q V$ também foram investigados. Utilizou-se a adaptação do questionário de Wallace e cols. $(2000)^{23}$ que avalia a gravidade da disfagia orofaríngea por meio do auto-relato dos sintomas. A investigação da QV se deu por meio do European Dysphagia Group Questionnaire. Oitenta e sete pacientes com CCP tratados com cirurgia e radioterapia ou quimioterapia e radioterapia concomitante fizeram parte do estudo. Constatou-se que 50,6\% dos pacientes apresentaram disfagia orofaríngea, sobretudo para alimentos sólidos (72,4\%). Cerca de $60 \%$ dos pacientes evitavam comer com outras pessoas e $37 \%$ se sentiam envergonhados no momento das refeições. A presença de disfagia foi maior entre os que foram submetidos à glossectomia total e quimiorradioterapia. Houve a necessidade de suporte nutricional em $57,1 \%$ dos pacientes; $20,3 \%$ apresentaram desnutrição e $51 \%$ relataram uma diminuição na sua $Q V$ atribuída à disfagia ${ }^{22}$.

Cinquenta e cinco pacientes submetidos à quimioterapia e radioterapia simultânea para - tratamento de tumores malignos localmente avançados na região de cabeça e pescoço foram avaliados a fim de investigar a capacidade para retomar a alimentação por via oral após o tratamento. Em um período médio de 17 meses, 25 pacientes $(45 \%)$ desenvolveram disfagia grave necessitando do uso de gastrostomia por mais de 3 meses. Após - tratamento, dos 33 pacientes submetidos ao estudo da deglutição (Modified Barium Swallow), 12 pacientes (36\%) apresentavam disfagia grave e aspiração silenciosa. Treze (39\%) desenvolveram disfagia requerendo suporte nutricional enteral prolongado adicional a ingestão oral. Durante o tratamento, grande parte dos pacientes teve severa perda de peso $(0-21 \mathrm{~kg})$ possivelmente devido à mucosite e alimentação por tubo de gastrostomia ${ }^{24}$.

A incidência de disfagia tem sido descrita como entre $50-60 \%$ em estudo com pacientes com CCP pós-tratamento $(25,5)$. Para avaliar a $Q V$, utilizou-se $\circ$ questionário da Universidade de Washington e o Hospital Anxiety and Depression. A gravidade da disfagia foi investigada por meio de videofluoroscopia da deglutição e Escala de desempenho. A qualidade de vida encontrouse diminuída após tratamento, em especial nos pacientes com disfagia moderada e grave. As funções de deglutição e fala, assim como lazer e desfiguração, foram as variáveis mais afetadas 5 .

\section{Qualidade de vida em deglutição}

A Organização Mundial de Saúde (OMS) define a QV como um conceito amplo, que engloba a saúde física, estado psicológico, nível de independência, relacionamento social e as relações com característica do meio em que indivíduo está inserido ${ }^{26}$.

A QV pode ser gravemente afetada pelo CCP e seu tratamento, uma vez que as interações sociais e emocionais estão intimamente ligadas à integridade estrutural e funcional dos órgãos envolvidos ${ }^{27}$. Dor/ desconforto, ansiedade e depressão foram relatadas mais frequentemente por pacientes recém-diagnosticados para CCP. A adição da disfagia, percepção do estado de saúde e dor contribuíram substancialmente para a perda de peso pré-tratamento, assim como o estágio avançado do tumor, como constatado por Lango e cols. $(2014)^{28}$.

Em geral, 40\% dos pacientes concordaram com as afirmações: "Eu não como tanto quanto eu costumava fazer", "Quase todos os dias não me importo se como ou não "," Eu estou raramente com fome " e " Eu não gosto de comer mais ". Dos 159 pacientes avaliados, 58\% teve perda de peso, atribuindo sua ingestão diminuída e perda de apetite principalmente a disfagia ${ }^{28}$.

Maurer e cols. $(2011)^{29}$ investigaram o impacto da disfagia na $Q V$ de 35 pacientes com carcinoma de células escamosas localmente avançado na região de cabeça e pescoço. Destes, $80 \%$ recebeu quimioterapia concomitante. Foram aplicados os 
questionários EORTC QLQ - C30 e EORTC H\&N 35 pré, peri e pós tratamento. Antes da terapia, $46 \%$ dos pacientes relatou dificuldades em deglutir e, ao final do tratamento, $86 \%$ apresentou este mesmo problema. Doze meses após o fim do tratamento, $15 \%$ ainda sofria deste sintoma. Observou-se maiores complicações alimentares nos pacientes que foram submetidos à quimioterapia concomitante, aumentando a incidência e a gravidade da disfagia $^{29}$.

As experiências da alimentação vivenciadas ao longo do tempo por pacientes com câncer tratados com radioterapia e quimioterapia concomitante têm demonstrado impacto na $Q^{6}$. Dezessete pacientes foram observados e entrevistados antes, durante e após o tratamento. Grande parte dos entrevistados relatou problemas mínimos na deglutição antes do tratamento, porém piora acentuada durante e após a radioterapia, sem um reestabelecimento da função. Apesar dos efeitos colaterais começarem a regredir após 6 meses, a deglutição permaneceu prejudicada, levando a mudanças importantes nas relações familiares, sociais e no estilo de vida.

Para muitos, a refeição simbolizava uma fonte de prazer, tais como desfrutar as comidas favoritas, experimentar novos alimentos, planejar as refeições e cozinhar. Além disso, a alimentação traduzia-se em um momento de aproximação com familiares e amigos, socialização. Imediatamente após o tratamento, o constrangimento durante as refeições era comum devido aos ruídos durante a mastigação, tosse, à ingestão exclusiva de alimentos pastosos e - sentimento de vergonha por não ser capaz de comer no mesmo ritmo que os outros. Houve grande variação na forma como estas pessoas se sentiam afetadas, restringindo o contato social, enquanto outros continuaram com os comportamentos sociais de antes ${ }^{6}$.

Os efeitos da radioterapia e da quimioterapia concomitante sobre a deglutição demonstram ser relevantes na $Q V$. A $Q V$ em deglutição foi avaliada por meio do Quality of Life in Swallowing Disorders Questionnaire (SWAL-QOL) em 110 pacientes com CCP constatando uma redução em todos os parâmetros avaliados para todos os pacientes, mesmo 6 e 12 meses após o término do tratamento $^{30}$. O questionário avaliou onze domínios: alimentação como um fardo, medo, desejo de se alimentar, comunicação, duração da alimentação, saúde mental, frequência de sintomas, social, seleção de alimentos, fadiga e sono. Em especial, alguns aspectos como o desejo e a duração ao comer, fardo e seleção de alimentos apontaram ter relevância para os pacientes, fragilizando sua saúde mental. Pacientes com tumores primários avançados (T4) tiveram os piores resultados para os parâmetros seleção de alimentos, comunicação e social. Quando o SWAL-QOL foi associado ao tipo de tratamento, os parâmetros mais afetados foram a deglutição como um fardo, fadiga e comunicação (30). Pacientes com disfagia moderada ou grave apresentam pior $Q V$ do que aqueles sem disfagia. Realizou-se uma análise retrospectiva com 73 pacientes que se queixavam de disfagia após a radioterapia primária (RT), quimioradioterapia, e RT pós-operatória de tumores malignos de cabeça e pescoço. A QV foi avaliada com os questionários da Universidade de Washington e Hospital Anxiety and Depression constatando que estes estão expostos à maior ansiedade e depressão, resultando na diminuição da ingestão de alimento devido à dificuldade em comer e o isolamento social ${ }^{5}$.

\section{CONSIDERAÇÕES FINAIS}

A disfagia é uma dificuldade que vai além do ato de nutrir-se e envolve aspectos emocionais, físicos e socioculturais, podendo acarretar em complicações fatais. É um sintoma comum em pacientes com câncer, em especial para o câncer de cabeça e pescoço, podendo ocorrer devido a doença e/ou modalidade de tratamento persistindo durante e/ou após o término do tratamento. A frequência e gravidade da disfagia dependerão do estágio e localização do tumor, quando envolvidos em estruturas que participem da deglutição, e o tratamento utilizado. Pacientes com tumores em estágios mais avançados e localizados na faringe tendem a apresentar piora na função da deglutição.

Investigar a qualidade de vida em deglutição pode complementar as avaliações clínicas e instrumentais e identificar aspectos específicos sobre o bem-estar do paciente que não foram por elas contemplados. 
A literatura tem demostrado que de fato a disfagia gera mudanças, como nos hábitos alimentares, prazer ao comer, perda de peso, relacionamento social e estado psicológico. Mais estudos são necessários englobando pacientes com diversos tipos de câncer e o impacto dos distúrbios da deglutição sobre sua qualidade de vida.

\section{AGRADECIMENTOS}

Às Instituições Universidade do Estado da Bahia, Escola Bahiana de Medicina e Saúde Pública, Universidade Federal da Bahia e a Fundação de Amparo à Pesquisa do Estado da Bahia (nRED0025/2014) e aos programas PICIN/UNEB (N BOL1715/2015) e PIBIC/FAPESB ( $N^{\circ}$ BOL1344/2014; $N^{\circ}$ BOL1330/2014), referente às bolsas de iniciação científica.

\section{CONTRIBUIÇÕES DOS AUTORES}

Carrera M, Medrado ARAP, Martins GB, Lima HR participaram da Concepção do projeto ou análise e interpretação dos dados; Costa AS e Marques RSO participaram da redação do artigo ou revisão crítica relevante do conteúdo intelectual autores; Carrera M, Medrado ARAP, Martins GB e Lima HR participaram da revisão e aprovação da versão final. Carrera $M$ é responsável por todos os aspectos do trabalho na garantia da exatidão e integridade de qualquer parte da obra.

\section{CONFLITOS DE INTERESSES}

Nenhum conflito financeiro, legal ou político envolvendo terceiros (governo, empresas e fundações privadas, etc.) foi declarado para nenhum aspecto do trabalho submetido (incluindo mas não limitandose a subvenções e financiamentos, conselho consultivo, desenho de estudo, preparação de manuscrito, análise estatística, etc).

\section{REFERÊNCIAS}

1. Brasil. Ministério da Saúde. Instituto Nacional de Câncer José Alencar Gomes da Silva, Coordenação de Prevenção e Vigilância. Estimativa 2016: incidência de câncer no Brasil. Rio de Janeiro. RJ. 2015.

2. Raber-Durlacher JE, Brennan MT, Verdonck-De Leeuw IM, Gibson RJ, Eilers JG, Waltimo T et al. Swallowing dysfunction in cancer patients. Support Care Cancer. 2012;20(3):43343. doi: $10.1007 / \mathrm{s} 00520-011-1342-2$

3. McHorney CA, Bricker DE, Kramer AE, Rosenbek JC, Robbins J, Chignell KA, et al. The SWAL-QOL outcomes tool for oropharyngeal dysphagia in adults: I. Conceptual foundation and item development. Dysphagia. 2000; 15(3): 11 5-21. doi: 10.1016/i.ctrv.2009.08.008
4. Gaspar MDRDF, Pinto GDSA, Gomes RHS, Santos RS, Leonor VD. Avaliação da qualidade de vida em pacientes com disfagia neurogênica. Rev. CEFAC. 2015;17(6): 193945.

5. Nguyen NP, Frank C, Moltz CC, Vos P, Smith HJ, Karlsson U et al. Impact of dysphagia on quality of life after treatment of head-and-neck cancer. Int J Radiat Oncol Biol Phys. 2005;61(3):772-8. doi: 10.1016/i.ijrobp.2004.06.017

6. Patterson JM., McColl E, Wilson J, Carding P, Rapley T. Head and neck cancer patients perceptions of swallowing following chemoradiotherapy. Support Care Cancer. 2015;23(12):3531-8. doi: 10.1007/s00520-015-2715-8

7. Furkim AM, Mattana A. Fisiologia da deglutição orofaríngea. In: Ferreira LP, Befi-Lopes DM, Limongi SCO, editores. Tratado de Fonoaudiologia. $1^{a}$ edição - São Paulo: Roca; 2004. P. 212-216.

8. Furkim AM, Silva RG. Programa de reabilitação em disfagia neurogênica. São Paulo: Frontis, 1999.

9. Logemann JA, Larsen K. Oropharyngeal dysphagia: pathophysiology and diagnosis for the anniversary issue of Diseases of the Esophagus. Dis Esophagus. 2012;25(4): 299304. doi: $10.1111 /$ i.1442-2050.2011.01210.x

10. Ribeiro LMM. Deglutição: processo normal e patológico. Monografia [Especialização em Motricidade Oral]. Londrina. Paraná: Centro de Especialização em Fonoaudiologia Clínica; 2000.

11. Marchesan IQ. Deglutição: diagnostico e possibilidades terapêuticas. In: Fundamentos em Fonoaudiologia. São Paulo: Guanabara-Koogan; 1998. P. 51-58.

12. Postma DS, Pillsbury HC. Pathophysiology and evaluation of swallowing disorders. In: Dobie RA, Pillsbury HC, Postma DS, Lanie RB. Otolaryngologic approach to swallowing Didsorders. American Academy of Otolaryngology-Head and Neck Surgery Foundation, INC 1984.

13. Manikantan K, Khode S, Sayed SI, Roe J, Nutting CM, Rhys-Evans $\mathrm{P}$ et al. Dysphagia in head and neck cancer. Cancer Treat Rev. 2009;35(8):724-32. doi: 10.1016/i. ctrv.2009.08.008

14. Murphy BA. Approaches to supportive care. In: Bernier J, editor. Head and neck cancer: multimodality management. Springer; 201 1.p.255-266. doi: 10.1007/978-1-44199464-6 17

15. Furia CLB. Disfagias mecânicas. In: Ferreira LP, Befi-Lopes DM, Limongi SCO, editores. Tratado de Fonoaudiologia. $1^{a}$ edição - São Paulo: Rocca; 2004. P. 386-404.

16. Rosenthal DI, Lewin JS, Eisbruch A. Prevention and treatment of dysphagia and aspiration after chemoradiation for head and neck cancer. J Clin Oncol. 2006;24(17):2636- 


\section{3. doi: $\underline{10.1200 / J C O .2006 .06 .0079}$}

17. Logemann JA, Rademaker AW, Pauloski BR, Lazarus CL, Mittal BB, Brockstein B et al. Site of disease and treatment protocol as correlates of swallowing function in patients with head and neck cancer treated with chemoradiation. Head Neck. 2006; 28(1):64-73. doi: 10.1002/hed.20299

18. Nguyen NP, Vos P, Moltz CC, Frank C, Millar C, Smith HJ et al. Analysis of the factors influencing dysphagia severity upon diagnosis of head and neck cancer. Br J Radiol. 2008;81(969):706-10. doi: 10.1259/bir/98862877

19. Pauloski BR, Rademaker a W, Logemann J a, Stein $D$, Beery $Q$, Newman L et al. Pretreatment swallowing function in patients with head and neck cancer. Head Neck. 2000;22(5):474-82. doi: 10.1002/1097-0347(200008)22:5<474::AIDHED6>3.0.CO;2-I

20. Cohen EE, Blair E, Vokes EE, Haraf DJ. Characteristics Associated With Swallowing Changes After Concurrent Chemotherapy and Radiotherapy in Patients With Head and Neck Cancer. Arch Otolaryngol Head Neck Surg. 2008;134(10):1060-5. doi: 10.1001/archotol.134.10.1060

21. Rosenthal DI, Mendoza TR, Chambers MS, Asper JA, Gning I, Kies MS et al. Measuring head and neck cancer symptom burden: The development and validation of the $M$. D. Anderson symptom inventory, head and neck module. Head Neck. 2007;29(10):923-31. doi: 10.1002/hed.20602

22. García-Peris P, Parón L, Velasco $C$, de la Cuerda C, Camblor M, Bretón I et al. Long-term prevalence of oropharyngeal dysphagia in head and neck cancer patients: Impact on quality of life. Clin Nutr. 2007;26(6):710-7. doi: 10.1016/i.clnu.2007.08.006

23. Wallace KL, Middleton S, Cook IJ. Development and validation of a self-report symptom inventory to assess the severity of oral-pharyngeal dysphagia. Gastroenterology. 2000; 1 18:678-87. doi: 10.1016/S0016-5085(00)70137-5

24. Nguyen NP, Moltz CC, Frank C, Vos P, Smith HJ, Karlsson $U$ et al. Dysphagia following chemoradiation for locally advanced head and neck cancer. Ann Oncol. 2004;1 5(3):383-8. doi: 10.1093/annonc/mdh 101

25. Gillespie MB, Brodsky MB, Day TA, Lee FS, Martin-Harris B. Swallowing-related quality of life after head and neck cancer treatment. Laryngoscope. 2004; 11 4(8):1362-7. doi: $10.1097 / 00005537-200408000-00008$

26. WHOQOL Group. The World Health Organization Quality of Life Assessment (WHOQOL): position paper from the World Health Organization. Soc Sci Med. 1995;41(10):1403-9. doi: 10.1016/0277-9536(95)00112$\underline{K}$

27. Graeff A, Leeuw JR, Ros WJ, Hordijk GJ, Blijham GH,
Winnubst JA et al. Long-term quality of life of patients with head and neck cancer. Laryngoscope. 2000; $110(1): 98-106$. doi: $10.1097 / 00005537-200001000-00018$

28. Lango MN, Egleston B, Fang C, Burtness B, Galloway T, Liu J et al. Baseline health perceptions, dysphagia, and survival in patients with head and neck cancer. Cancer. 2014;1 20(6):840-7. doi: 10.1002/cncr.28482

29. Maurer J, Hipp M, Schäfer C, Kölbl O. Dysphagia: Impact on quality of life after radio(chemo)therapy of head and neck cancer. Strahlentherapie und Onkol. 2011 ; 187(1 1):7449. doi: $10.1007 / \mathrm{s} 00066-011-2275-\mathrm{x}$

30. Silveira M, Dedivitis R, Queija D, Nascimento P. Quality of Life in Swallowing Disorders after Nonsurgical Treatment for Head and Neck Cancer. Int Arch Otorhinolaryngol. 2014 ; 19(01):046-54. doi: 10.1055/s-0034-1395790 